\title{
Philosophy Meets Entertainment: Designing an Interactive Virtual Philosopher
}

\author{
Xuan Wang ${ }^{1,2}$, Eng Tat Khoo ${ }^{3}$, Sanath Siriwardana ${ }^{1}$, \\ Horathalge Iroshan ${ }^{1}$, and Ryohei Nakatsu ${ }^{1,2}$ \\ 1 Keio-NUS CUTE Center, Interactive and Digital Media Institute, \\ National University of Singapore \\ \{wangxuan, hssiriwardana, idmhorat, elenr\}@nus.edu.sg \\ 2 NUS Graduate School for Integrative Sciences and Engineering, Singapore \\ 3 Eon Reality, Singapore \\ engtat@eonreality.com.sg
}

\begin{abstract}
To many people, philosophy seems to be a difficult and daunting subject. Our research seeks to make the esoteric philosophical ideas and concepts more accessible to people in the modern world, and make philosophy learning an entertaining activity by allowing people to directly interact with virtual philosophers from the past. With Artificial Intelligence technology, we have created a virtual philosopher that can automatically respond to user's input in natural language text. It is hoped that the added interactivity can help to increase the appeal of philosophical subject to the users, and the users can have a better idea of the philosophy after an entertaining experience talking with the virtual philosopher. In this paper, we share our considerations for designing the system, the system architecture, and our preliminary user study on the interaction with the virtual philosopher.
\end{abstract}

Keywords: Virtual philosophers, interactive philosophy, artificial intelligence, conversational agent.

\section{Introduction}

To many people, philosophy seems to be a difficult and daunting subject. Though philosophical concepts are hard to grasp, learning about them do benefits us 4]. Traditionally, the main channel for the public to learn about philosophy is through reading books. Recently, the advent of digital technology is reshaping the way people learn about things [12]. Passive media such as books are gradually losing its appeal to the new interactive digital media - people now possess a higher level of digital literacy, and are more inclined to acquire knowledge through the modern networked and digital media.

Observing this trend, we started to look into this question: is there a way to make philosophy less intimidating and more interesting, so that even today's born-digitals would like to know more about it? Drawing inspirations from the field of Edutainment, which makes learning more fun by adding new media 
elements such as video games, our research tries to leverage people's digital literacy, and bring traditionally esoteric philosophical ideas and concepts into an entertaining context, in order to make them more appealing and more accessible to the general public.

New entertainment technology needs to offer an active experience in order for users to reach enjoyment [8. Based on this theory, we have designed a system that makes philosophy learning interactive. Rather than trying to get a grip of the ideas through thousands of lines in the books or sit back and watch a video, people can directly speak 'face to face' to the philosophers through the activity of online chat. We hope to promote philosophy learning, or at least dispel the common stereotype about philosophy, by creating an interactive system with fun interface but also deep meaning behind. We are interested to see how people respond to such system, and whether such a system can actually be used in the real world as a new way for learning and interacting with philosophy.

In this paper, we present our system, iSage, a virtual conversational agent that models philosophical knowledge and impersonates sages in the past. We discuss the design considerations of creating a virtual philosopher, and present the architecture of our prototype system. We share the lessons learnt from making the system and the user testing, and discuss the future directions of this research.

\section{Design Considerations}

As reasoned above, one of the issues with philosophical books is its huge volume and esoteric appearance. In fact, there has been endeavours in making traditional texts more friendly to the modern readers by means of adding modern language interpretations and even caricatures. Efforts have also been made to bring the thoughts and even the life experiences of the sages into digital media in the production of films and cartoons series. Electronic resources are also available on the internet in the form of online databases and ebooks. However, in these situations, the interaction between the user and the medium is still one-way, and the user still largely remains as a passive receiver rather than an active explorer.

Speaking of sages, most people naturally feel a distance towards them, thinking they are lofty ideals that are beyond normal people's understanding. Modern philosophers see this problem and try to relate philosophy in people's everyday life and explain it in ways that are easy to understand. Askphilosophers.org is a web site that gathers talents and knowledge of trained philosophers to answer questions from the general public 1 . The site has attracted people at all stages of life and successfully proves that philosophy can be explained in intelligible ways and be used to help people. However, this site needs to be managed by people, and it takes time to get the answers as the answers are all given manually by the real philosophers.

${ }^{1}$ http://www.askphilosophers.org/ 
Different from the approach of askphilosophers.org, we want to develop an automatic system that is interactive and appealing to users. We have identified the following three characteristics that are necessary:

- Interactivity. Users should not be just passive receivers, but instead active enquirers. Active experience is one of the important conditions that lead to enjoyment 8 . More freedom should be given to user to freely explore things in which they are interested. It does not necessarily have to follow a linear or logical path (as in the case of book reading or film watching), but a path of interest.

- Short and fast interaction. The system should be easy to get access to and readily available. Users do not need to take the time and read a huge book, but can interact and get an idea in a very short time.

- Easy-to-use interface. No prior knowledge is required to interact with the system. In other words, we should take advantage of the digital literacy that people already own, and present it in a medium that is familiar to most of the people today.

The above considerations lead to the decision of making a virtual recreation of sages in history with a mobile interface. We want to let the sages speak for themselves, share their life stories, reveal their admirable charisma, and become advisors or even friends to people. People can easily use the system to talk freely to the sages, whether for a casual chat or to seek insights of the profound philosophy. In this way, it tailors to the specific needs of each individual and offers a personalized dialogue experience with a virtual sage of choice.

\section{Related Work}

Chat bots, also known as conversational agents, have appeared long ago. One of the first chat bots, Eliza, was invented as early as in the 1960s [15]. It is a simulation of a Rogerian psychotherapist that can talk to patients. Since then, numerous chat bots have been created 2 . However, the focus of such chat bots is to use various techniques to disguise as a human being [7. Generic answers are given when the question is out of bounds of the knowledge base, which often does not offer real help to the user. On the other hand, virtual conversational agents have also been used for online help, as we can see on many companies' websites, and they have been used in museums as museum guides [6]. They are used for learning as well, for example, to learn a foreign language [2]. Researchers have also built a chat bot for people to access the Quran, the central religious text of Islam 11]. Most of these systems usually have no understanding of the input, but solely rely on pre-programmed questions and answers. As a result, the output is confined within the scope of trained patterns, which means limited ability to handle user request.

Compared to these chat bots, our virtual philosopher differs in two important ways:

2 e.g. Cleverbot, http://www.cleverbot.com/ and Jabberwacky, http://www.jabberwacky.com/ 
1. Understanding: The normal chat bots usually rely on pattern matching to provide an answer. In cases when a template cannot be matched, tricks such as asking the question back are used to make it appear like understanding. However, for users who would like to seek advice from the virtual agent, this does not offer any help, and can sometimes be frustrating. Therefore, in our system, we attempt to handle unseen inputs by syntactic and semantic analysis, and relate the input to the knowledge base entries via semantic closeness.

2. Authenticity: Unlike many of the chat bots which are arbitrarily created characters that handle small talks and are just for fun, our system represents a real existed person. Moreover, it represents the renowned historical giants, to whom people naturally have much respect and expectations. This also presents great technical challenges to model the philosopher's thinking accurately.

\section{Creating a Virtual Confucius}

Among the many influential schools of thought, we decided to choose Confucianism as our first attempt in interactive philosophy. This is mainly because we are a lab based in Asia where Confucianism is very influential. Though this Asian philosophical system is more than 2500 years old, it is still being studied nowadays, and classic Confucian ideas such as filial piety, respect for elders and man's relation to his society are still very relevant to the modern society.

\subsection{Building the Knowledge Base}

The first step of creating a virtual Confucius is to gather large amounts of data and build a knowledge base. Because this is a highly specialized domain of knowledge, we have to work together with the domain experts. We collaborated with researchers specializing in Confucius philosophy in our university. They helped us to gather data from classical texts: the Analects, the Mencius, the Book of Rites and the Classic on Filial Piety. These four texts constituted a major part of educational curriculum for children in China 13.

The whole set of Confucius knowledge base is divided into three broad categories, according to the nature of the statements. We believe that due to the distinctive nature of the information in each of these categories, they should be treated separately both in terms of the knowledge representation as well as retrieval methods. The first category is domain specific factual knowledge, including information about Confucius as an individual, for example, his age, his date of birth, his hometown, etc., as well as introductory statements about the figures appearing in Confucius's responses (mostly Confucius's disciples). We gather this information from the earliest reliable historical text of Shiji by Sima Qian (ca. 110B.C.). As information in this category is straightforward factual knowledge, we choose to store them in Artificial Intelligence Markup Language (AIML) scripts [14, which is good for handling such query. For instance, if the 
user asks "who is Yan Hui", Confucius's reply will directly be taken from the scripts saying, "Yan Hui is my favourite disciple".

The second category is the major part of the knowledge base, which contains all Confucius's sayings and ideas, collected from the above mentioned classical texts. Moreover, paragraphs in these texts are separated into short but selfsustained statements. For instance, the opening passage of the Analects becomes three entries in our database: 1. Is it not pleasant to learn with a constant perseverance and application? 2. Is it not delightful to have friends coming from distant quarters? 3. Is he not a man of complete virtue, who feels no discomposure though men may take no note of him? In this way, a total of 2069 entries were collected and stored in an SQL database.

For each sentence, the domain experts tagged it with 1-3 topic(s). The reason for this manual work is due to the special nature of the domain knowledge, which often contains implicit meanings that are impossible to deduce from the literal understanding of the sentence using natural language processing technology. A total of 309 topics were identified. These topics are used later in our algorithm to compare the relatedness of the entry with the user inputs. These topics were further tagged with specific senses in the context of Confucius's teaching. This is necessary because many of the topics in this domain have particular meanings, and literal word level tagging cannot convey the precise meaning and would lead to confusion. For example, the topic 'way' in Confucian context means 'a course of conduct', rather than direction or path. Therefore, for the topic 'way', it is tagged as 'way\# $\# \# 5$ ', which is the fifth meaning of the word 'way' in the WordNet lexical database [1. We chose to use WordNet because of its comprehensiveness and its ontology that makes possible the comparisons of semantic similarities between words. By tagging the sentences with the topics, it effectively transforms them into points in a high dimensional space, where each dimension represents one topic.

WordNet is a general ontology. Though it is good in handling everyday concepts, it is not sufficient to cover all the concepts in the Confucian knowledge base. Therefore, it is necessary to create a separate knowledge base with domainspecific terminologies. For instance, names of classical texts from which Confucius often quotes, and Confucian terminologies such as Junzi (the morally ideal man in Confucian philosophy) and Ren (benevolence, or the 'way of being' that of an exemplary person), etc, need to be captured and denoted specifically. As these words do not have semantic meanings, entries related to these terminologies are tagged with the literal words and stored in the SQL database. Some sentences from the second category are also tagged with keywords if they contain certain terminology. A total of 319 sentences were tagged with such keywords.

\subsection{System Architecture}

The core of the system is a virtual sage thinking engine, which analyses user inputs, calculates and extracts the most appropriate answer. A front-end mobile application is used to interact with the virtual philosopher. Figure 1 shows the architecture of the system. 


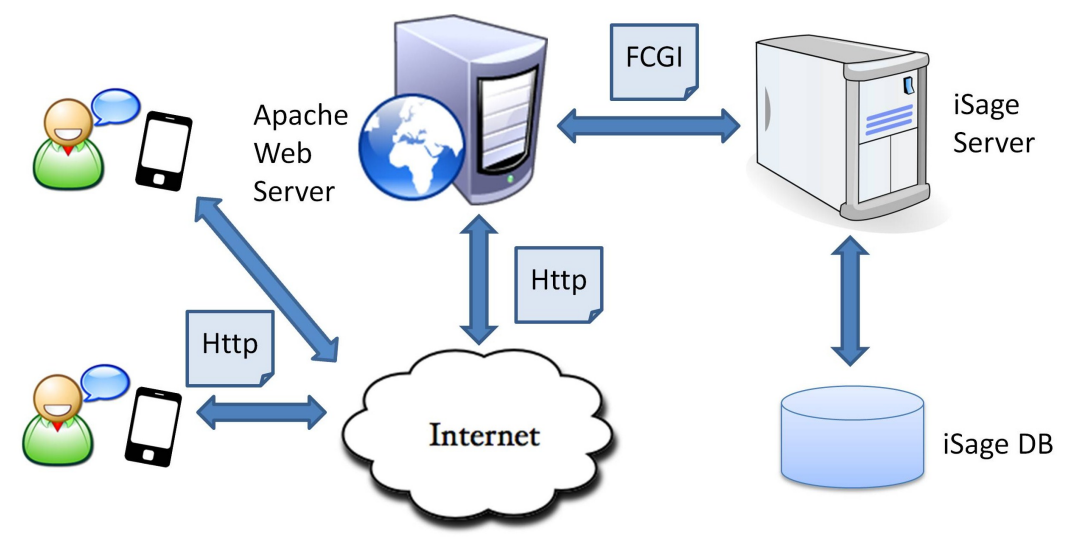

Fig. 1. Architecture of iSage system

The virtual sage thinking engine consists of three different modules: a pattern matching module, a keyword matching module, and a semantic closeness matching module. Figure 2 shows an overall composition of the virtual sage thinking engine. Each of the modules is designed to handle different part of the knowledge base, as described before.

The Pattern Matching Module. The pattern matching module contains pre-scripted templates and their matching rules. This is a technique commonly used in many chat bots, which has been proven to be very effective in providing good responses as long as the question falls within the pre-scripted set. It also helps to make the bot converse more like a person. We have used a modified A.L.I.C.E. AIML [14 knowledge base in our system. Unrelated templates are deleted, and many of the templates are modified to be more Confucius-like with the help of the domain experts, according to the information collected in the knowledge base building phase. The quality of an answer from this module is assessed by how much the pattern matches the input. If there isn't a readily available answer for the input, the answer selection process will go onto the next stage.

The Semantic Closeness Matching Module. We certainly cannot predict all the questions that will be possibly asked and prepare an answer for each. Especially for the case of building a virtual Confucius, doing so would require very good knowledge on the subject and would be extremely time-consuming. Therefore, sophisticated natural language processing and reasoning are necessary to automatically extract the answer for unseen input. In the semantic closeness matching module, a series of NLP techniques are employed to find important words in the sentences as well as their meanings, and then match them with a relevant answer based on their semantic closeness. There are three stages: important words identification, topics identification, and database entry selection. As 


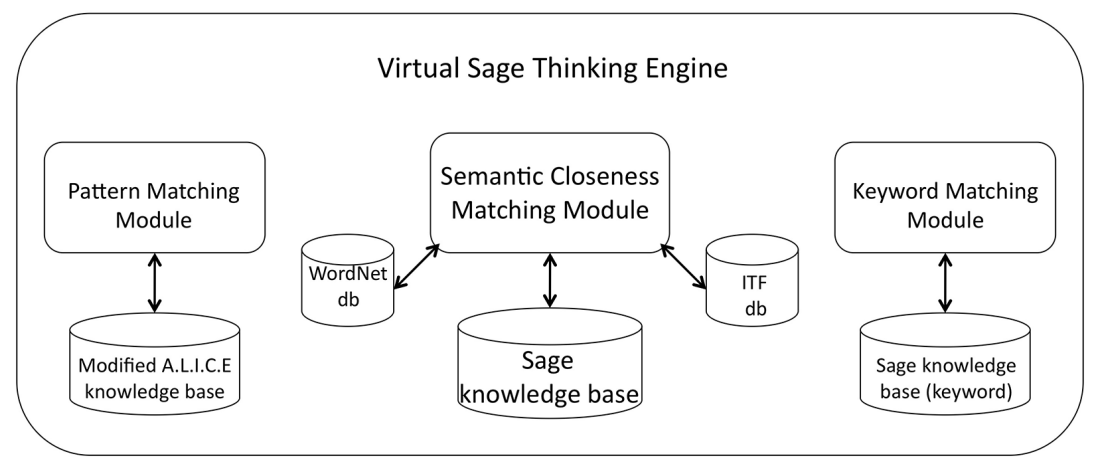

Fig. 2. Composition of the virtual sage thinking engine

mentioned before, a lexical database called WordNet 1 is used to find semantic relations of English words.

In the important words identification stage, the user input is first fed into a parser [5] that analyses the grammatical structure of the sentence. The last noun of each noun phrase is selected as the important word [3]. Furthermore, we employ Inverse Term Frequency (ITF) to find additional important words, based on the idea that the more frequent word such as 'the', 'and' do not contribute much to the real meaning of the sentence, whereas the less frequent words, 'loyal', 'conflict', for example, are more important. An ITF database is built by calculating the frequency of appearance of each word in a large corpus. Combining the results from the above two methods, a list of important words can be extracted from the user input. Furthermore, the user input is passed through a Word Sense Disambiguation (WSD) module [10 to get the meaning of these words in the context. This information, together with the important words selected, is used to compare the semantic closeness against a list of pre-defined topics in the sage knowledge base. This is done using a WordNet-based similarity module WordNet::Similarity 9], which computes a semantic similarity score for any given pair of words. Each important word selected is compared with the list of topics. The final value for each topic is based on the summation of the similarity scores with each important word. Scores that are below a certain value are discarded to reduce noise. After this step, the input sentence is digitized as a point in the high dimensional space of topics. It can be represented as a vector $\boldsymbol{v}$ :

$$
\boldsymbol{v}=\sum_{i=1}^{m} \varphi\left(\operatorname{sim}\left(w_{i}, p_{1}\right)\right) \boldsymbol{t}_{\mathbf{1}}+\cdots+\sum_{i=1}^{m} \varphi\left(\operatorname{sim}\left(w_{i}, p_{n}\right)\right) \boldsymbol{t}_{\boldsymbol{n}}
$$

where $m$ is the number of important words extracted from the input sentence, $n$ is the total number of topics, $\boldsymbol{t}_{\boldsymbol{n}}$ is the basis vector representing the $n t h$ topic, $\operatorname{sim}(w, p)$ is the similarity score between word $w$ and topic $p$, and

$$
\varphi(x)= \begin{cases}x & \text { if } \mathrm{x} \text { is greater than threshold } \\ 0 & \text { otherwise }\end{cases}
$$


Finally, the process enters the database entry selection stage. Here, the user input sentence is represented as a vector in a high dimensional space constructed by the list of topics. Similarly, each database entry is also determined as a vector in this high dimensional space, according to the taggings by the domain experts. The fitness of a sentence as an output to a certain input is assessed by the Euclidean distance between the two points. The database entry with the closest distance is selected as the output, if the distance is below a certain threshold value.

The Keyword Matching Module. In the Keyword Matching Module, a simple string comparison between the keywords in the database with the user input is carried out. Each matched word would increase the matching score for the sentence by 1 . If the highest sentence score is more than a threshold, the corresponding entry is deemed as suitable for output. If, at this stage, still no suitable answer can be extracted, the system will ask the user to rephrase the question, or to change the topic.

User inputs go through a layered process and are analysed by the above three modules sequentially. The suitability of output is evaluated by computing a quality score. These three methods handle different types of inputs and thus complement each other. Figure 3 shows a simplified flow diagram of the system.

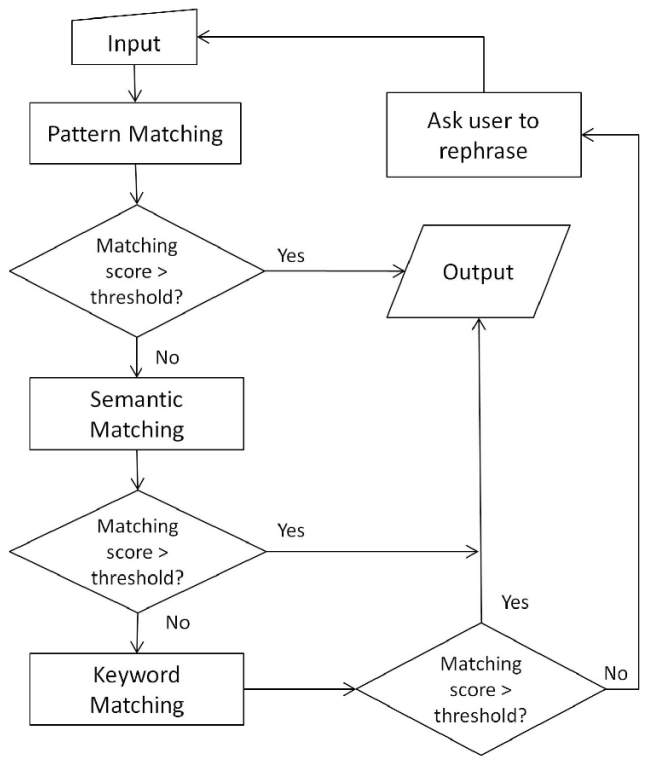

Fig. 3. A simplified flow diagram of the virtual sage thinking engine 


\subsection{Example Usage}

Following are some example outputs given by this algorithm.

- Question: What is love?

- Answer: Love commences with respect as its basis. To neglect respect is to leave affection unprovided for.

- Question: How should I treat my parents?

- Answer: A son of all comprehensive virtue serves his parents as he serves Heaven.

- Question: I am worried.

- Answer: If a man takes no thought about what is distant, he will find worries near at hand.

- Question: Tell me something about the Book of Poetry.

- Answer: It is said in the Book of Poetry: be always studious in order to be in harmony with the ordinances of Heaven, you will certainly get for yourself much happiness.

\subsection{The Application}

We have created a Android application, iSage Confucius, which allows people to talk to the virtual Confucius on their smart phones by typing their questions. After receiving the query, the mobile application sends the request to the iSage server, where the virtual Confucius thinking engine is located. The server processes the incoming request, and returns the answer given by our algorithm. The answer is then displayed on the user's phone as Confucius's reply, as if the user is having a real conversation with the sage. Figure 4 shows two screenshots of the application.

To make it more fun for users, we also added a function for sharing the answer to social networks such as Twitter and Facebook. Users can also rate the answer if they think it is a very profound answer, a funny answer, or a bad answer. They can also go to another page to see the questions asked by other people and the answers given by Confucius, ranked according to the query time or the ratings, as is shown on the right in Figure 4. This is to add more diversity to the application other than a robot that simply answers. The rating function serves research purpose as well, because we can get a better idea of the quality of the system through human feedback.

\section{User Evaluations}

To see people's reactions to this system as a new way to interact with philosophy, and to gather feedbacks to improve the system, a pilot user testing was conducted. 


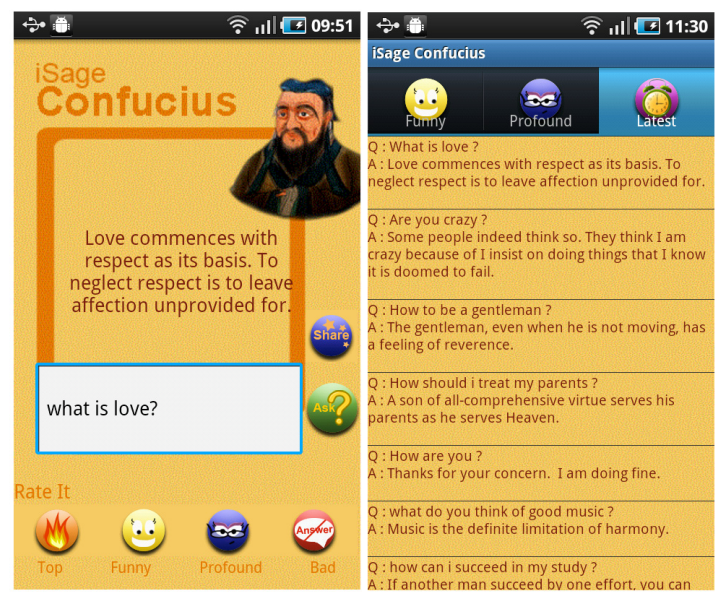

Fig. 4. Screenshots of the iSage Confucius application

\subsection{Participant Demography}

Twenty participants were recruited for the study (15 male and 5 female). All of them are under the age of 35 , and nineteen of them have experiences or are very familiar with mobile applications. The participants come from a variety of ethnic groups, but are all Asian except one. 7 of them indicated that they knew nothing about Confucius's philosophy, 11 said they knew "a little", and 2 had a moderate amount of knowledge. About their interests in Confucianism, 2 indicated that "I have great passion for it", 12 "would like to learn about it if possible", 4 are "not interested, but do not mind to know more", and 1 participant indicated "not interested at all". When asked about the reasons that prevent them from knowing more about the Confucian culture, 11 of them said it is because of a lack of time. Besides reasons like "too difficult to understand", or "just don't like it", other reasons reported by the participants include "not enough interest to look for information on my own, and not enough reported in the media". This implies that an application that can offer fast and entertaining interaction might be useful and attractive to them.

\subsection{Procedure}

The participants were first informed that he/she was going to interact with a virtual Confucius, which is a computer program that tries to mimic the real Confucius. They could use this mobile application to converse with Confucius, and they could ask any questions they like. The participants were asked to interact with the system for about 10 minutes and fill in a questionnaire afterwards to report their experience with the application. 
We did not impose any constraints on questions to be asked and restricted them to the topics that are more suitable to be answered by Confucius, partly because we are curious what users have to say to a virtual Confucius, but also because we would like to see the system's ability to handle arbitrary questions and user's reactions to the answer (be it good or bad) given.

\subsection{Results}

In the questionnaire, the participants were asked to rate their experiences and opinions based on a 5-point Likert scale. The responses to the questions are presented as bar charts in Figure 5. Many of the participants were excited to use the system, and all participants except one indicated that they enjoyed interacting with the application. $60 \%$ of the participants said they became more interested in Confucian culture and would like to know more. Some comments from the participants are "it is very user friendly and interesting", "the idea and the vision is interesting", "answered some of my questions pretty logically, and seem to comprehend the nature of my question".

17 out of 20 participants agreed that interacting with the application is more fun than reading a book. However, when asked whether they think it can achieve the same effect as reading a book, most of them hold a conservative view: only 7 agreed or strongly agreed. This is not surprising, as book reading has always been considered the traditional and proper way to acquire knowledge, especially for this kind of philosophical knowledge which requires one to take time to delve

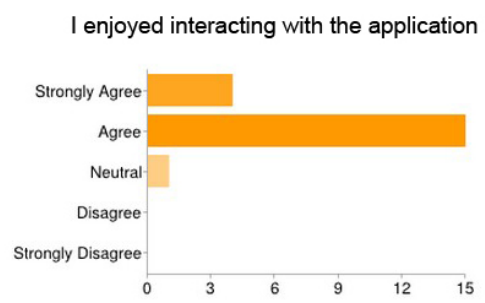

(a)

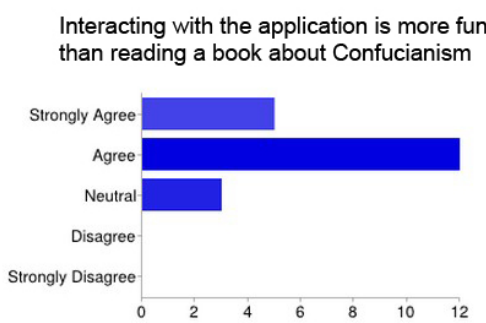

(c)

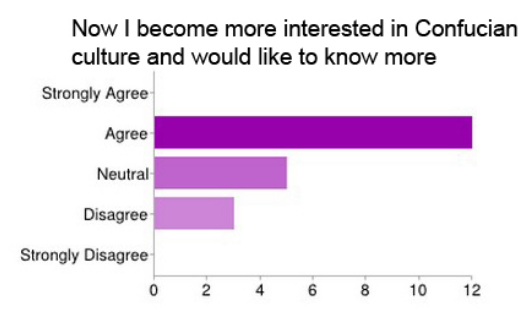

(b)

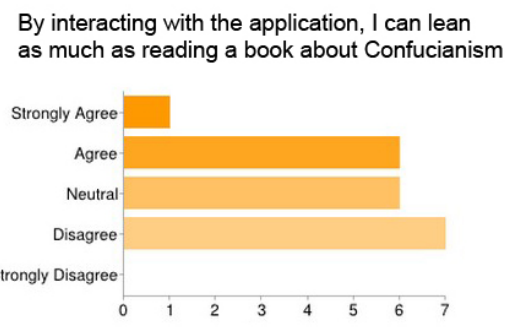

(d)

Fig. 5. Results of the questionnaire from the user study 
into, to reflect and distil. However, for some people, they may never pick up a book and read the Analects, but if they are presented with an entertaining interactive system, they are much more likely to try it out and play with it. This is also one of the important motivations and goals of building this system - to make philosophy learning more enjoyable and to introduce Confucianism to more people.

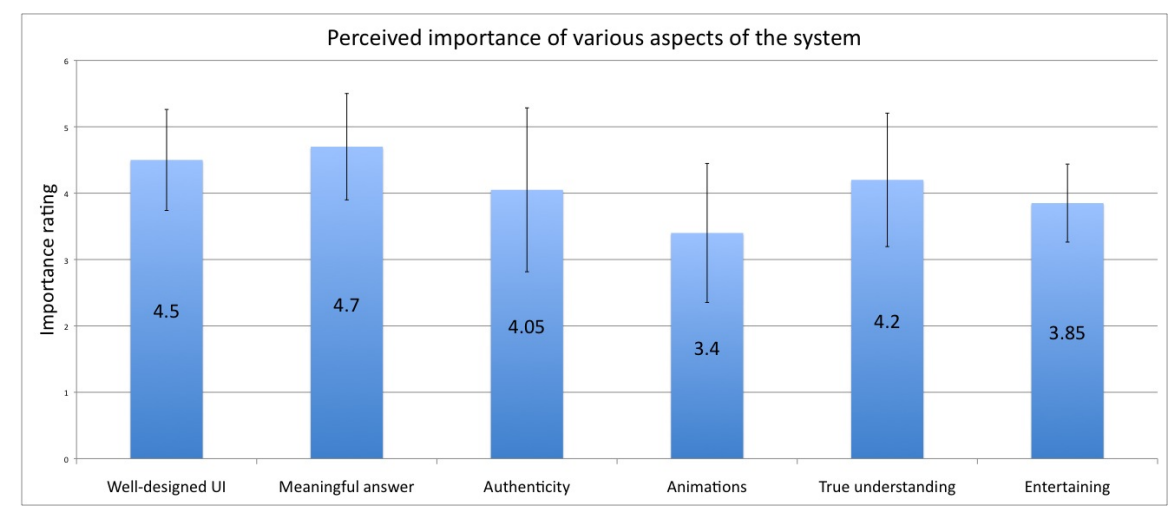

Fig. 6. Participants' perceived importance of various factors of the system

In addition, the participants were asked to give a rating of the importance of various factors of the system based on a 5-point Likert scale, with 1 being the least important and 5 being the most important. The candidate options are: well-designed UI, meaningfulness of the answer, authenticity (i.e. whether it truly represents the opinion of Confucius), animations of Confucius showing emotions, true understanding (note that this is different from meaningfulness, as the system can have no understanding of the user but still output a sensible answer, as in the case of many chatterbot systems), and entertainment value. Participants were explained in more detail if they were not sure what the option means. The results of the average ratings are shown in Figure 6] Quality of the answer, or the meaningfulness of the answer is deemed as the most important aspect of the system, and plays an important role in providing a satisfying experience. Participants care relatively less about whether the system truly understands them or not, but they expect the answers to be meaningful. In terms of 'authenticity', participants hold a more varied view, with some thinking it is very important, but some considering it not so important. UI design is also considered an important factor, as people expect to have an easy-to-use system. Entertainment value is somewhat important, but much less so as compared to the answer's meaningfulness. Other interaction modalities, for example, the use of animations, are deemed as the least important among all the factors. 


\section{Conclusion and Future Work}

In this paper, we have presented our efforts in making philosophy interactive by creating a virtual philosopher. A mobile application, iSage Confucius, is developed. We hope it can allow more people to be able to interact with the valuable pool of knowledge and draw upon the ancient wisdom in an easy and entertaining way. From our pilot user testing, we can see that this mode of interaction is liked by users, but there are many more that needs to be improved. Rather than constraining our research in a lab environment, we want to push our research results into the wild and benefit the general public. At the same time, we hope that real world usage of our application can provide us valuable insights and information to improve our system.

From the results of the pilot user study, we can see that people have high expectations of the answers given by a virtual sage. Our current algorithm sometimes gives unrelated answers, and lacks the ability to comprehend words without semantic meanings such as people's names. In future works we should focus on designing better AI algorithms. One of the possible improvements is to look up information on the internet for words that it does not understand, and use the relevant information for further inference. For example, if the user asks about Obama, the system should search on the internet and find Obama is a political leader, then look in the knowledge base for politics-related views such as how to govern a country. In addition, we want to test the effectiveness of the current structure of the system by conducting controlled studies where people use varied versions of the system containing only part of the modules in the virtual sage thinking engine.

Apart from improving the system, we also want to know the effects of this application. This work is done under the original intention of promoting philosophy and let it be known and liked by more people, we should test whether this goal can be achieved. However, unlike mathematics or physics where there can always be a definite answer, philosophy is very abstract and often subjective. Thus, it is very difficult to assess how much knowledge about philosophy people gain after using the system. Evaluation of such kind of systems is an interesting and important question to be addressed in future works.

To make a virtual recreation of great sages in the past is clearly not an easy task, and we are just beginning the first step. We believe that philosophy learning can be made into an entertaining activity using interactive new media technology.

Acknowledgements. This research is supported by the Singapore National Research Foundation under its International Research Centre @ Singapore Funding Initiative and administered by the IDM Programme Office.

\section{References}

1. Fellbaum, C.: WordNet: An Electronic Lexical Database. MIT Press, Cambridge (1998) 
2. Fryer, L., Carpenter, R.: Bots as Language Learning Tools. Language Learning \& Technology 10(3), 8-14 (2006)

3. Huang, Z., Thint, M., Qin, Z.: Question classification using head words and their hypernyms. In: Proceedings of the Conference on Empirical Methods in Natural Language Processing, Morristown, NJ, USA, pp. 927-936. Association for Computational Linguistics (October 2008)

4. Kindersley, D.: The Philosophy Book. Dorling Kindersley Ltd. (2011)

5. Klein, D., Manning, C.D.: Accurate unlexicalized parsing. In: Proceedings of the 41st Annual Meeting on Association for Computational Linguistics - ACL 2003, pp. $423-430(2003)$

6. Kopp, S., Gesellensetter, L.: A Conversational Agent as Museum Guide - Design and Evaluation of a Real-World Application. In: Panayiotopoulos, T., Gratch, J., Aylett, R.S., Ballin, D., Olivier, P., Rist, T. (eds.) IVA 2005. LNCS (LNAI), vol. 3661, pp. 329-343. Springer, Heidelberg (2005)

7. Mauldin, M.L.: Chatterbots, Tinymuds, and the Turing Test Entering the Loebner Prize Competition. In: Machine Translation, pp. 16-21 (1994)

8. Nakatsu, R., Rauterberg, M.: A New Framework for Entertainment Computing: From Passive to Active Experience. In: Kishino, F., Kitamura, Y., Kato, H., Nagata, N. (eds.) ICEC 2005. LNCS, vol. 3711, pp. 1-12. Springer, Heidelberg (2005)

9. Pedersen, T.: WordNet::Similarity - Measuring the Relatedness of Concepts. In: Proceedings of the Nineteenth National Conference on Artificial Intelligence (AAAI 2004), San Jose, CA. Number Patwardhan 2003, pp. 1024-1025 (2004)

10. Pedersen, T., Kolhatkar, V.: WordNet::SenseRelate::AllWords: a broad coverage word sense tagger that maximizes semantic relatedness. In: Proceedings of the North American Chapter of the Association for Computational Linguistics - Human Language Technologies 2009 Conference, Boulder, CO., pp. 17-20. Association for Computational Linguistics (2009)

11. Shawar, B.A., Atwell, E.: Accessing an Information System by Chatting. In: Meziane, F., Métais, E. (eds.) NLDB 2004. LNCS, vol. 3136, pp. 407-412. Springer, Heidelberg (2004)

12. Tapscott, D.: Growing up digital: The rise of the Net Generation. McGraw-Hill (June 1998)

13. Theodore de Bary, W.: Confucian Education in Premodern East Asia. In: Tu, W.-M. (ed.) Confucian Traditions in East Asian Modernity, pp. 21-38. Harvard University Press, Massachusetts (1996)

14. Wallace, R.: The elements of AIML style. Alice AI Foundation (2003)

15. Weizenbaum, J.: ELIZA-a computer program for the study of natural language communication between man and machine. Communications of the ACM 9(1), 36-45 (1966) 